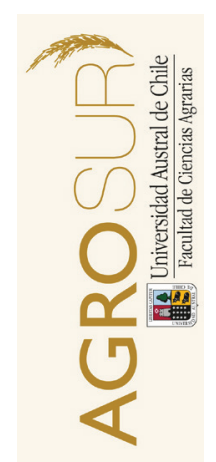

\title{
Influencia de la época de parto y recuento de células somáticas sobre el rendimiento productivo y reproductivo para Overo Colorado en la Región de Los Lagos
}

\author{
Influence of calving season and somatic cell score on reproductive \\ and productive performance of Overo Colorado in Los Lagos Region
}

\author{
Mujica, F. ${ }^{a^{*}}$, Uribe, $\mathrm{H}^{b}{ }^{b}$, Rosas, J. ${ }^{a}$ \\ ${ }^{a}$ Instituto de Producción Animal, Universidad Austral de Chile. Casilla 567, Valdivia, Chile. \\ ${ }^{b}$ Departamento Producción Animal, Universidad de Chile. Av. Santa Rosa № 11315, Santiago, Chile.
}

\begin{abstract}
A B S T R A C T
The Overo Colorado breed, is a dual-purpose cattle breed; selection process over time has led it to acquire adaptation traits and rusticity and becoming an alternative to southern Chilean dairy production systems based on grazing. This study was done using milk recording data from the Cooperativa Agrícola y Lechera de Frutillar Ltda. (CAFRA) considering 1,800 lactations, of Overo Colorado cattle at the province of Llanquihue, in Los Lagos Region of Chile. The general objective was to describe productive and reproductive performance of the cows, considering calving season and somatic cell score (RCS). The hypothesis of this study was that calving season and somatic cell score, do not influence dairy production and reproductive performance. Using linear multiple regression models, least square means were obtained for the variables of interest: milk yield and composition, and calving interval (LIP). The descriptive results showed milk yield mean at 4,467 $\mathrm{kg} \mathrm{cow}^{-1}$. Fat and protein percentage mean values were found at $3.63 \%$ and $3.4 \%$, respectively. The proposed linear models determined that calving season and RCS, had significant effects on yield and LIP. Regarding milk yield, lactations initiated in winter and autumn were significantly more productive than those commenced in other season; LIPs were lower for winter and autumn calving seasons. For fat percentage, the lactations started in summer obtained higher values, and protein percentage was favorable for lactations starting in spring. Meanwhile, milk production and fat percentage decreases as RCS and LIP increased.
\end{abstract}

\section{RESUMEN}

Overo Colorado es considerada una raza bovina de doble propósito; selección genética a través del tiempo la ha provisto de características de adaptación y rusticidad, convirtiéndose en una alternativa para los sistemas lecheros basados en praderas. Este estudio utilizó registros de control lechero de la Cooperativa Agrícola y Lechera de Frutillar Ltda. (CAFRA) considerando 1.800 lactancias de vacas Overo Colorado de la provincia de Llanquihue en la Región de los Lagos. El objetivo fue identificar la capacidad productiva y reproductiva, considerando la época de parto y el recuento de células somáticas (RCS). La hipótesis planteada fue que la época de parto y el recuento de células somáticas, no influyen sobre los rendimientos productivos lecheros y reproductivos. A través de modelos de regresión lineal múltiple, para las variables analizadas, se obtuvo el promedio mínimo cuadrado de ellas. Los resultados descriptivos presentaron una media para la producción láctea de $4.467 \mathrm{~kg} \mathrm{vaca}^{-1}$, de 3,63\% para grasa y 3,4\% para proteína. Los modelos propuestos, determinaron que la época de parto y el RCS tuvieron un efecto significativo sobre los rendimientos productivos y el lapso interparto (LIP). En producción de leche, lactancias iniciadas en invierno y otoño fueron significativamente más productivas; los LIPs fueron menores para las mismas épocas. Con respecto al porcentaje de grasa, las lactancias iniciadas en verano obtuvieron los mayores valores, y para el porcentaje de proteína fue favorable la época de primavera. A medida que aumenta el RCS disminuye la producción láctea, el porcentaje de grasa y aumenta el LIP.

Palabras clave: leche, bovinos Overo Colorado, Sur de Chile.

\section{INTRODUCCIÓN}

La raza Overo Colorado, también conocida como Clavel Alemán, fue traída a Chile por los colonizadores alemanes a fines del siglo 19. Son animales considerados inicialmente doble propósito, de color rojo y blanco, de tamaño mediano, rústicos, con bajas perdidas de individuos por patologías. Es una raza con gran versatilidad, adaptándose a las fluctuaciones del mercado y climáticas, permitiendo al productor escoger entre carne o leche, o desarrollar ambas, logrando buenos resultados económicos (FAO, 2007).

Aliaga (2004) señala que, en el rubro de producción de carne, un novillo de entre 18 y 20 meses alcanza un 
peso de $500 \mathrm{~kg}$ y a los 24 meses de $550 \mathrm{~kg}$. Según datos obtenidos por Uribe y Smulders (2004) la producción láctea ajustada a los 305 días es de $5.044 \mathrm{~kg}$ para esta raza.

La reproducción es un proceso biológico fundamental para un sistema productivo, por ello, deben ser considerados los diversos factores que pueden afectar la preñez (Wattiaux, 1999). La mayoría de las vacas lecheras tienen la capacidad de reproducirse a intervalos de 12 a 13 meses, consiguiendo 10 meses de lactancia, de este modo la eficiencia reproductiva se liga a la producción láctea (Gasque, 2008).

Cuando el sistema de producción es en base a praderas con partos concentrados desde fines de invierno a comienzos de primavera se habla de un sistema estacional, el cual tiene por objetivo la concentración de un gran número de partos, con la finalidad que coincida de mejor manera la curva de crecimiento de la pradera con los requerimientos nutricionales del rebaño en lactancia (González, 2008).

En el mercado de la leche, no solo la cantidad de litros producidos es importante, en la actualidad los productores reciben un bono por calidad de la leche. El Consorcio Lechero (2013) señala que para Chile, el contenido de sólidos en la leche posee en promedio valores de $3,77 \%$ para materia grasa y $3,35 \%$ para proteína. Uno de los factores que afecta el contenido de sólidos, es el nivel de células somáticas presentes en la leche. Cuando los niveles de recuento de células somáticas (RCS) son altos, comienzan a aparecer alteraciones, y se puede ver una disminución de algunos de sus componentes como el porcentaje de materia grasa, calcio y magnesio; otros componentes aumentan como el cloro y sodio (Carrillo y Vidal, 2002).

Philpot y Nickerson (2000), señalan que el RCS se define como la cantidad de células somáticas por mililitro de leche. Además indican que en cuartos con mastitis, el RCS puede ascender a 1 millón $\mathrm{mL}^{-1}$. Butendieck (1997), indica que la presencia de mastitis arroja un alto RCS, y se relaciona con los cambios que provoca en la composición química de la leche, alterando propiedades organolépticas y durabilidad, reduciendo su calidad.

Otros factores que influyen sobre la concentración de los componentes sólidos de la leche, son: raza, edad, etapa de lactancia y estado nutricional interno, los cuales son clasificados como factores endógenos; y como factores exógenos se encuentra el medio ambiente donde se desarrolla el animal. Altas temperaturas pueden afectar de manera indirecta el consumo de materia seca (especialmente fibra), provocando una reducción del volumen de leche y la concentración de grasa. La concentración de proteína también se ve afectada, debido a una reducción de la síntesis proteica ruminal. Por su parte, temperaturas cercanas o bajo $0{ }^{\circ} \mathrm{C}$, aumentan el costo de mantención disminuyendo el aporte de energía a la glándula mamaria (Manterola, 2007).
El Consorcio Lechero (2013) señala que el contenido de sólidos en la leche sigue un patrón estacional bastante definido. Observándose que los contenidos de proteína aumentan notoriamente durante la primavera $(3,42 \%)$ con respecto al invierno $(3,29 \%)$, y al mismo tiempo, la materia grasa sufre un descenso en primavera $(3,61 \%)$ en comparación al verano $(3,82 \%)$ y otoño $(4,05 \%)$ provocado por un insuficiente contenido de fibra en la pradera.

La hipótesis planteada para el rebaño del presente estudio es que "para la raza Overo Colorado la época de parto y el recuento de células somáticas, no influyen en los rendimientos productivos lecheros y reproductivos".

Este estudio tiene como objetivo general identificar, a partir de los datos disponibles y procesados, la capacidad productiva y reproductiva considerando la época de parto y el recuento de células somáticas de vacas Overo Colorado, pertenecientes a un rebaño ubicado en la Región de Los Lagos.

\section{MATERIAL Y MÉTODO}

Los datos utilizados se obtuvieron de registros pertenecientes al control lechero realizado por la Cooperativa Agrícola y Lechera de Frutillar Ltda. (CAFRA). Se evaluaron lactancias terminadas entre los años 1999 y 2014, obteniéndose un total de 1.800 observaciones. El predio en estudio se ubica en la Región de Los Lagos, provincia de Llanquihue, específicamente en la comuna de Puerto Varas. El clima presente en esta zona es templado oceánico, presentando precipitaciones a lo largo de todo el año (ODEPA, 2014).

\section{El rebaño}

El rebaño lechero está compuesto por vacas de la raza Overo Colorado, con una incorporación promedio de un $10 \%$ de la raza Holstein Friesian. El productor del presente estudio pertenece a la Asociación Nacional de Criadores de la Raza Overo Colorado (ANACOC). Con respecto a la reproducción del predio, se realiza inseminación artificial, utilizando semen congelado de toros provenientes de Alemania, también es utilizado el semen de hijos de estos toros. El sistema productivo se basa en el pastoreo, por ello los animales se encuentran en la pradera todo el año. Las praderas destinadas a la alimentación son naturales y artificiales, ambas con fertilización, de estas praderas se obtiene silo pre-marchito. Durante la lactancia, como estrategia, se añade a la dieta alrededor de 1,5 kg promedio de concentrado por vaca al día que se suministra durante la ordeña.

\section{Procesamiento de datos}

Se construyó una planilla Excel basada en los registros facilitados por CAFRA. Los datos ingresados 
fueron editados, producto de lo cual, se obtuvieron y utilizaron los datos finales de: producción total por lactancia, RCS y porcentaje de materia grasa y proteína, también se incluyeron los días en lactancia. La información reproductiva, para este estudio, el lapso interparto (LIP), fue calculada con las fechas de parto.

Para el estudio de los datos se utilizó el programa estadístico Statistical Analisys System (SAS Copyright (C), 2002 - 2003). A través del módulo PROC MEANS, disponible en el programa estadístico SAS, se realizó el análisis de estadística descriptiva simple (promedio y desviación estándar) de las variables productivas y reproductivas. Para cuantificar la relación entre época de parto, recuento de células somáticas, variables productivas y reproductivas se utilizó regresión lineal múltiple. Con el módulo PROC GLM (modelos lineales generalizados), del programa SAS, se estimaron los promedios mínimos cuadrados. Se incluyeron en los modelos estadísticos todas las variables disponibles en la base de datos, con el objeto de corregir el posible efecto de estas en las variables dependientes. Se utilizó la prueba de Fisher ( "f") para determinar el nivel de significancia que las variables e interacciones tienen en el modelo propuesto.

Previo al análisis de las lactancias completas, fueron excluidas vacas con solo un registro de lactancia, debido a que no entregan datos del LIP. Además se eliminó de la base de datos información cuya veracidad es dudosa, a modo de evitar algún error en los cálculos, como: días en leche menores a 122 días o mayores a 488 y lapsos interparto mayores a 630 días, esto último debido a que se presume, que existe un parto o aborto de por medio.

En el predio, los partos ocurren durante todo el año, por consiguiente, para facilitar el manejo de la información se agruparon las fechas de parto, asignándole un número según época: primavera, verano, otoño e invierno. Además se realizó una categorización asignán- dose una sigla para: la producción láctea, porcentaje de grasa y proteína, recuento de células somáticas y lapso interparto; se crearon 4 grupos para cada variable, como se observa en el Cuadro 1.

Para el análisis en los modelos estadísticos empleados, en la variable Número Ordinal de Parto (NOP), se realizó una agrupación de animales con NOP igual o mayor a seis (NOP 6). En cuanto al RCS, se formaron 4 categorías, donde la presencia de células somáticas, en la categoría 1 es baja o normal hasta 4 que es muy alto.

\section{Modelos estadísticos}

Se establecieron diferentes modelos estadísticos, para inferir el efecto de la época de parto y el RCS, sobre las variables dependientes de interés (variables productivas y lapso interparto). Las variables incluidas en los modelos estadísticos fueron: época de parto (EPARTO), días en leche (DEL), número ordinal de parto (NOP), lapso interparto (CLIP), producción láctea (LECHE), porcentaje grasa (PGR), porcentaje proteína (PPRO) y recuento células somáticas (RCS), no se consideraron las interacciones que no eran significativas. Los modelos estadísticos utilizados en el estudio se presentan a continuación:

Modelo producción láctea $(\mathrm{kg})$ :

$\mathrm{Y}_{\text {ijklmnop }}=\mu+\mathrm{EPARTO}_{\mathrm{i}}+\mathrm{RCS}_{\mathrm{j}}+\mathrm{CLIP}_{\mathrm{k}}+\mathrm{NOP}_{1}+\mathrm{PGR}_{\mathrm{m}}+$ $\mathrm{PPRO}_{\mathrm{n}}+\mathrm{DEL}_{\mathrm{o}}+\left(\mathrm{RCS}^{*} \mathrm{PGR}\right)_{\mathrm{jm}}+\mathrm{e}_{\mathrm{ijklmnop}}$

Modelo para porcentaje de grasa:

$\mathrm{Y}_{\mathrm{ijklmno}}=\mu+\mathrm{EPARTO}_{\mathrm{i}}+\mathrm{RCS}_{\mathrm{j}}+\mathrm{NOP}_{\mathrm{k}}+\mathrm{LECHE}_{1}+\mathrm{PPRO}_{\mathrm{m}}+$ $\operatorname{CLIP}_{n}+$ DEL $_{o}+e_{i j k l m n o}$

Modelo para porcentaje de proteína: $\mathrm{Y}_{\mathrm{ijklmno}}=\mu+\mathrm{EPARTO}_{\mathrm{i}}+\mathrm{RCS}_{\mathrm{j}}+\mathrm{NOP}_{\mathrm{k}}+\mathrm{LECHE}_{1}+\mathrm{PGR}_{\mathrm{m}}+$ $\operatorname{CLIP}_{\mathrm{n}}+\mathrm{DEL}_{\mathrm{o}}+\mathrm{e}_{\mathrm{ijklmno}}$

Cuadro 1. Agrupaciones y niveles asignados para las variables épocas de parto, producción láctea, porcentaje de grasa y proteína y lapso interparto.

Table 1. Groups and levels assigned to calving season, milk yield, fat and protein percentage and calving interval variables.

\begin{tabular}{lccccc}
\hline Variable & Código & \multicolumn{4}{c}{ Agrupaciones } \\
\hline Época de parto & EPARTO & Sept - Oct - Nov. & Dic - Ene - Feb. & Mar - Abr - May. & Jun - Jul -Ago. \\
Producción láctea (kg) & LECHE & $1.000-2.500$ & $2.500-5.000$ & $5.000-7.500$ & $7.500-10.000$ \\
Grasa (\%) & PGR & $<3,0$ & $3,01-3,5$ & $3,51-4,0$ & $>4,01$ \\
Proteína (\%) & PPRO & $<2,5$ & $2,51-3,0$ & $3,01-3,5$ & $>3,51$ \\
LIP (días) & CLIP & $183-294$ & $294-405$ & $405-516$ & $516-630$ \\
RCS (miles cel $\mathbf{~ m L}^{-1}$ ) & RCS & $<100-200$ & $200-500$ & $500-1.000$ & $>1.000$ \\
\hline
\end{tabular}


Modelo para lapso interparto (días):

$\mathrm{Y}_{\mathrm{ijkmn}}=\mu+\mathrm{EPARTO}_{\mathrm{i}}+\mathrm{RCS}_{\mathrm{j}}+\mathrm{NOP}_{\mathrm{k}}+\mathrm{LECHE}_{1}+\mathrm{DEL}_{\mathrm{m}}+$ $\left(\text { EPARTO }^{*} \mathrm{NOP}\right)_{\mathrm{jk}}+\mathrm{e}_{\mathrm{ijklmn}}$

Modelo para RCS (miles cel $m L^{-1}$ ):

$\mathrm{Y}_{\mathrm{ijklmno}}=\mu+\mathrm{EPARTO}_{\mathrm{i}}+\mathrm{NOP}_{\mathrm{j}}+\mathrm{CLIP}_{\mathrm{k}}+\mathrm{LECHE}_{1}+\mathrm{PGR}_{\mathrm{m}}+$ $\mathrm{PPRO}_{\mathrm{n}}+\mathrm{e}_{\mathrm{ijklmno}}$

\section{RESULTADOS Y DISCUSIÓN}

En el Cuadro 2, se exponen los resultados del análisis descriptivo de las variables en estudio, señalando la media y desviación estándar obtenidos para cada una de ellas.

Los animales en estudio se encuentran todo el año en pastoreo, con suplementación de ensilaje pre-marchito en la época de invierno. Durante la lactancia, la incorporación de concentrado es menor a $15 \%$ del total de materia seca de la dieta, esto favorece al porcentaje de grasa presente en la leche, obteniéndose un resultado de 3,63\% y 3,4\% de proteína.

Según lo señalado por Hazard (1997) quien indica que el factor nutricional afecta el contenido graso de la leche, raciones que consideran un alto suministro de concentrado ( $>60 \%$ ), o que se enfocan en aumentar la producción láctea, implican una disminución en el

Cuadro 2. Resultados de estadística descriptiva para las variables en estudio.

Table 2. Descriptive statistical results for variables under study.

\begin{tabular}{lrrr}
\hline Variable & \multicolumn{1}{c}{ N } & Media & $\begin{array}{c}\text { Desv. } \\
\text { Estándar }\end{array}$ \\
\hline Prod. láctea total $\left.{ }^{1} \mathbf{( k g}\right)$ & 1.676 & $4.467,87$ & $1.240,02$ \\
Prod. láctea: Primavera & 535 & $4.172,77$ & $1.208,24$ \\
Prod. láctea: Verano & 194 & $4.444,06$ & $1.159,41$ \\
Prod. láctea: Otoño & 537 & $4.671,10$ & $1.297,02$ \\
Prod. láctea: Invierno & 410 & $4.598,03$ & $1.169,12$ \\
Días en leche & 1.676 & 268,30 & 50,17 \\
No ordinal de parto $^{\circ}$ & 1.676 & 3,68 & 1,76 \\
\% Grasa & 1.676 & 3,63 & 0,37 \\
\% Proteína & 1.676 & 3,40 & 0,22 \\
LIP $^{2}$ (días) & 1.376 & 380,25 & 50,53 \\
RCS $^{3}$ (miles cel mL $^{-1}$ ) & 1.676 & 397,62 & 520,16 \\
\hline
\end{tabular}

${ }^{1}$ Produccion láctea total, sin estandarización a 305 días.

${ }^{2}$ Lapso interparto.

${ }^{3}$ Recuento de Células Somáticas. contenido de grasa. Por el contrario, dietas ricas en fibra efectiva permiten que aumente la concentración de materia grasa láctea.

Con respecto a la proteína láctea, Hazard (1997) menciona que es afectada de una manera más marginal con la manipulación dietética que el contenido de grasa. Algunos componentes nutricionales pueden hacer variar el contenido de proteína de la leche, como los suplementos que se entregan en la forma de grasa, o la entrega de un pobre contenido energético o proteico, pueden causar una baja de 0,3 puntos.

El promedio de los días de lactancia fue de $268,3 \pm$ 50,17 días (Cuadro 2), valor bajo si se toma en consideración que los días de lactación para bovinos productores de leche se estima alrededor de 305 días o 10 meses, con dos meses (periodo seco) para la recuperación apropiada del tejido mamario (Latrille, 1985).

En el Cuadro 2, se observa un resultado promedio de 12,5 meses (380,24 días) para el lapso interparto (LIP) en la raza OC, este resultado esta sobre los 365 días recomendados por Uribe y Lanuza (2006). La reproducción y la eficiencia reproductiva son vitales para el rubro lechero, la meta para mantener la eficiencia reproductiva y la rentabilidad de una explotación lechera, es logrando un lapso interparto promedio de 13 meses (Fricke, 2009).

El promedio del RCS (Cuadro 2) para el rebaño en estudio fue de 397,62 miles cel $\mathrm{mL}^{-1}$, resultado que se encuentra en la categoría medio según lo indicado por Butendieck (1997). El RCS tiende a ser menor en rebaños de alta producción de leche, principalmente por: la alta dilución y por el hecho de ser de mayor producción, generalmente reciben un manejo sanitario superior, por lo tanto, se le aplican medidas de prevención y control de mastitis (Philpot y Nickerson, 2000).

\section{Análisis de los modelos estadísticos para el efecto Época de parto y Recuento de Células Somáticas (RCS)}

Se analizó el efecto de las variables independientes, Época de parto y RCS, sobre variables dependientes: producción de leche (kg), porcentaje de grasa y proteína presente en la leche, recuento de células somáticas y la variable reproductiva lapso interparto. La interpretación de resultados se basó en la comparación de promedios mínimos cuadrados (PMC).

\section{Efecto de la época de parto}

En el Cuadro 3 se observa que la época de parto tuvo un efecto significativo en la producción láctea, porcentaje de grasa, porcentaje de proteína, LIP y RCS.

Lactancias iniciadas en invierno obtienen mayores producciones acumuladas de leche que lactancias iniciadas en primavera y verano. Por el contrario, se 
registra una menor producción de leche en lactancias iniciadas en verano y primavera. Esto se puede atribuir a que a mediados de otoño, ocurre una disminución en la disponibilidad de pradera, manteniéndose hasta la época invernal, afectando a todos los animales, independiente de la fecha de parto, considerando la eventual sequía estival, que limita la disponibilidad y calidad de las praderas, se obtiene que vacas con inicio de lactancia más tardía sean las más afectadas.

Las diferencias en producción láctea para cada época, pueden ser explicadas por un factor nutricional. En la época de primavera ocurre un repunte de la productividad y calidad de la pradera, en este caso, las lactancias iniciadas en invierno se vieron favorecidas por la primavera siguiente.

Para el porcentaje de grasa, los partos de verano alcanzaron un valor de 3,605\% siendo este superior al de otras épocas (Cuadro 3); se debe considerar que la alimentación del rebaño en estudio es realizada en base a pradera, por lo cual el efecto de la época del año sobre esta conlleva a modificaciones nutricionales. Considerando esto, el hecho de observar mayor porcentaje de grasa en la época de verano, se debería al aumento de los niveles de fibra disponibles en las praderas a medida que se avanza hacia los meses más secos. Pérez (2003) analizó las épocas de otoño y primavera, con resultados de 3,72\% y 3,71\% respectivamente, no observando diferencias significativas entre estas, al igual que en este estudio.

En un estudio de factores que influyen en los sólidos de la leche Valderrama (2011), obtuvo que en la Zona Sur del país, la concentración de grasa es mayor en verano, otoño e invierno. Un valor inferior se obtuvo para primavera, lo cual se explica por el alto consumo de pradera, que en esa época contienen un menor contenido de fibra. Klein (2003) indica que en primavera existe insuficiente fibra estructural para estimular una adecuada masticación y rumia; esto provoca fecas muy blandas, un $\mathrm{pH}$ ruminal marginalmente bajo y menor concentración de grasa en la leche.

Lactancias iniciadas en la época de primavera presentaron un mayor porcentaje de proteína $(3,48 \%)$, con respecto a las otras épocas de parto. La composición nutricional de la pradera es altamente variable durante el año, Klein (2003) señala que la concentración de nutrientes en la materia seca del forraje puede variar entre $13 \%$ y $30 \%$ de proteína cruda y entre 2,3 y 3,0 Mcal de energía metabolizable, entre el verano y primavera, respectivamente. Del mismo modo encontró valores para carbohidratos no estructurales (CNE) de 24,1 para primavera y 17,8 para verano, en el llano longitudinal de la Décima Región.

Manterola (2007) analizó diferentes factores nutricionales sobre el porcentaje de proteína láctea y observó que el consumo de energía es el factor más importante, ya que por cada MegaJoule extra de energía se aumenta en 0,03 unidades porcentuales de proteína. $\mathrm{Al}$ incrementarse el aporte de energía en la ración por aumento en los CNE se produce un aumento de la producción de leche y del porcentaje de proteína.

Valderrama (2011) señala que al considerar aspectos alimentarios sobre la proteína, la pradera tiene un efecto positivo, principalmente en la Zona Sur, debido a que en su estudio halló un aumento de proteína en primavera y otoño cuando hay más pastoreo. Lo señalado anteriormente concuerda con los resultados de este estudio, ya que las vacas $\mathrm{OC}$, son alimentadas durante todo el año con praderas.

En el Cuadro 3 se observa que la época de parto tuvo un efecto significativo sobre el LIP, lactancias iniciadas en primavera y verano arrojaron un mayor LIP, que lactancias iniciadas en otoño e invierno. Los LIPs hallados en este estudio están en general por debajo de los 392 días (12,9 meses), que según lo recomendado por Fricke (2009), es la meta que un productor debe alcanzar. Con respecto a esto, todos los LIP encontrados

Cuadro 3. Promedios mínimos cuadrados de producción láctea, porcentaje de grasa y proteína, lapso interparto y recuento de células somáticas, a través de épocas de parto.

Table 3. Least square means of milk yield, fat and protein percentage, calving interval and somatic cell count, across calving seasons.

\begin{tabular}{cccccc}
\hline & \multicolumn{4}{c}{ Promedios mínimos cuadrados } \\
\cline { 2 - 5 } & Prod. láctea (kg) & \% Grasa & \% Proteína & LIP $^{\mathbf{1}}$ (días) & RCS $^{2}$ (miles cel mL $^{-1}$ ) \\
\hline Primavera & $4.226,03^{\mathrm{b}}$ & $3,561^{\mathrm{b}}$ & $3,486^{\mathrm{a}}$ & $386,69^{\mathrm{a}}$ & $241,05^{\mathrm{b}}$ \\
Verano & $4.118,61^{\mathrm{b}}$ & $3,605^{\mathrm{a}}$ & $3,417^{\mathrm{bc}}$ & $386,97^{\mathrm{a}}$ & $289,75^{\mathrm{b}}$ \\
Otoño & $4.289,85^{\mathrm{a}}$ & $3,522^{\mathrm{b}}$ & $3,393^{\mathrm{c}}$ & $377,77^{\mathrm{b}}$ & $380,12^{\mathrm{a}}$ \\
Invierno & $4.349,49^{\mathrm{a}}$ & $3,516^{\mathrm{b}}$ & $3,422^{\mathrm{b}}$ & $380,02^{\mathrm{ab}}$ & $242,61^{\mathrm{b}}$ \\
\hline
\end{tabular}

Letras distintas dentro de columna indican diferencias significativas $(\mathrm{p}<0,05)$ según prueba "f". ${ }^{1}$ Lapso interparto. ${ }^{2}$ Recuento de Células Somáticas. 
en este estudio, para cada época de parto, se consideran adecuados.

En la época de verano se puede presentar un estrés calórico causado por la radiación solar, velocidad del viento, temperatura del aire y la humedad ambiental, esto puede afectar la reproducción, teniendo como respuesta una disminución de la intensidad del estro y -como consecuencia- una baja en la fertilidad (Córdova et al., 2008). El número de días que la vaca no está preñada, es la principal causa de un LIP prolongado y por consiguiente de problemas reproductivos.

González y Magofke (2007) señalan que en las últimas décadas las producciones (leche, grasa y proteína) en la raza Holstein Friesian se han incrementado, no obstante, en el mismo período ha existido un marcado deterioro en caracteres reproductivos. Hazard (1997) señala que al contrario de los animales de menor producción, las vacas con alto nivel productivo le cuesta más quedar preñadas. Durante los primeros 60 días de lactancia, los animales poseen una alta demanda de nutrientes, y éstos al no ser cubiertos afectan tanto a la producción como a la reproducción, debido a que la vaca no ovula. El rebaño OC de este estudio, como ya fue señalado, está catalogado como una raza doble propósito, por lo tanto sus niveles productivos son inferiores a los de la raza Holstein.

El RCS también se vio afectado por la época de parto, como se puede apreciar en el Cuadro 3, lactancias iniciadas en otoño poseen un RCS mayor $(380,12$ mil cel $\mathrm{mL}^{-1}$ ) que a lactancias iniciadas en otras épocas. Este resultado no coincide a lo señalado por Philpot y Nickerson (2000) quienes indican que, para los Estados Unidos, en los meses de verano, con condiciones de calor y humedad, aumenta la mastitis desencadenando un incremento de células somáticas, alcanzando un promedio nacional de $500.000 \mathrm{cel} \mathrm{mL}^{-1}$, siendo éste superior al promedio de los meses de invierno que van desde los 300.000 a los $350.000 \mathrm{cel} \mathrm{mL}^{-1}$.
Es importante considerar que bajos RCS se logran cuando las vacas se mantienen en ambientes higiénicos, secos y confortables, como las praderas bien manejadas. Las condiciones climáticas y el manejo en general son factores importantes dentro de un programa permanente sobre control de mastitis, cuyos principios deben aplicarse constantemente.

\section{Efecto del recuento de células somáticas}

En el Cuadro 4 se presentan los valores de los promedios mínimos cuadrados del efecto del RCS sobre las variables: producción láctea, porcentaje de grasa, porcentaje de proteína y lapso interparto; observándose que el RCS tiene un efecto significativo sobre éstas.

El Grupo 1, el cual es considerado dentro del rango normal $\left(<100.000-200.000 \mathrm{cel} \mathrm{mL}^{-1}\right)$, posee la mayor producción láctea, al contrario del grupo 3 y 4 $\left(>500.000 \mathrm{cel} \mathrm{mL}^{-1}\right.$ ) que obtuvieron una menor producción. Estos resultados coinciden con lo señalado por Philpot y Nickerson (2000), quienes estimaron pérdidas anuales de leche de $91 \mathrm{~kg}$ en la primera lactancia y $182 \mathrm{~kg}$ en lactancias posteriores cada vez que el RCS se duplicaba entre 50.000 y $800.000 \mathrm{~mL}^{-1}$. Con RCS superiores a los $200.000 \mathrm{~mL}^{-1}$ comienzan las pérdidas económicas, a medida que aumente el número de células somáticas, las perdidas en producción de leche y pérdidas económicas por lactancias aumentan, existiendo una relación inversa entre el RCS y la producción de láctea, esto es coincidente con lo encontrado en diferentes estudios como el de Alt (2005) y Pedraza et al. (2000).

Para el porcentaje de materia grasa presente en la leche (Cuadro 4), se observa que a mayor número de células somáticas el porcentaje de grasa disminuye, encontrándose diferencias significativas entre el Grupo 1, 2 y 3 con el Grupo 4, siendo este último el que posee un menor porcentaje de grasa. En promedio, aquellas vacas con un RCS inferior a $500.000 \mathrm{cel} \mathrm{mL}^{-1}$ (grupo

Cuadro 4. Promedios mínimos cuadrados de producción de leche, porcentaje de grasa y proteína y lapso interparto, para los distintos grupos de recuento de células somáticas.

Table 4. Least square means of milk yield, fat and protein percentage and calving interval, for different groups of somatic cell score.

\begin{tabular}{ccccc}
\hline & \multicolumn{4}{c}{ Promedios mínimos cuadrados } \\
\cline { 2 - 5 } Grupos (RCS $)$ & Producción láctea $(\mathbf{k g})$ & Grasa $(\%)$ & Proteína $(\%)$ & LIP $^{\mathbf{2}}$ (días) $^{\mathrm{y}}$ \\
\hline $\mathbf{1}$ & $4.464,32^{\mathrm{a}}$ & $3,586^{\mathrm{a}}$ & $3,405^{\mathrm{b}}$ & $375,03^{\mathrm{b}}$ \\
$\mathbf{2}$ & $4.275,42^{\mathrm{b}}$ & $3,588^{\mathrm{a}}$ & $3,399^{\mathrm{b}}$ & $380,51^{\mathrm{ab}}$ \\
$\mathbf{3}$ & $4.071,95^{\mathrm{b}}$ & $3,550^{\mathrm{a}}$ & $3,461^{\mathrm{a}}$ & $386,36^{\mathrm{a}}$ \\
$\mathbf{4}$ & $4.172,31^{\mathrm{b}}$ & $3,479^{\mathrm{b}}$ & $3,454^{\mathrm{a}}$ & $389,54^{\mathrm{a}}$ \\
\hline
\end{tabular}

Letras distintas dentro de columna indican diferencias significativas $(\mathrm{p}<0,05)$ según prueba " $\mathrm{f}$ ". ${ }^{1}$ Recuento de Células Somáticas. ${ }^{2}$ Lapso interparto. 
1 y 2) produjeron 0,109 unidades porcentuales más de materia grasa en la leche, que aquellas con un RCS $>1.000 .000 \mathrm{~mL}^{-1}$.

Philpot y Nickerson (2000) señalan que la leche normal posee un 3,5\% de grasa y con un alto RCS esta baja a 3,2\%. Los resultados encontrados por Pedraza et al. (2000), en su estudio de cambios en la composición láctea por efecto del incremento de células somáticas, concuerdan a lo obtenido en este análisis, donde se señala que existe una relación negativa entre la grasa presente en la leche y el rango de RCS.

De los resultados del Cuadro 4 , se puede deducir que con altos RCS existe un mayor porcentaje de proteína, lo cual difiere a lo observado por Philpot y Nickerson (2000), quienes indican que la leche con un alto RCS posee $3,56 \%$ de proteína, contrastando con leche de calidad normal $\left(<200.000 \mathrm{cel} \mathrm{mL}^{-1}\right)$ la cual posee 3,61\% de proteína, aludiendo a un efecto negativo sobre el porcentaje de proteína, provocado por un mayor RCS.

A raíz de los resultados obtenidos en su estudio, Pedraza et al. (2000) señala que el nivel de RCS que registran los animales, tiene una gran influencia sobre los sólidos no grasos. Natzke et al. (1965) y Haenlein et al. (1968) determinaron una correlación negativa de $-0,382$ entre sólidos no grasos y el RCS.

El estrés es uno de los factores medioambientales que más afecta la producción y reproducción animal. La presencia de mastitis subclínica, reflejado en un elevado RCS, provoca estrés en el animal, lo cual es perjudicial para el desempeño reproductivo de las vacas, ya que retrasa el primer estro, después del parto, aumentando los días de intervalo parto-primer servicio, disminuye la fertilidad, incrementa los días abiertos y servicios por concepción y acrecienta la susceptibilidad al aborto temprano, por lo cual el periodo entre partos se extiende de manera significativa (Córdova et al., 2008). Es importante mantener un registro del RCS, ya que la producción de leche es fruto de un evento reproductivo, y este podría estar deprimido como consecuencia de una mastitis subclínica reflejada en un elevado RCS. En los sistemas pastoriles, como es el caso de este estudio, su importancia es sustancialmente mayor que en estabulación, porque se busca ajustar los requerimientos alimenticios de la vaca con el crecimiento de la pradera.

Los PMC encontrados para la variable lapso interparto (LIP), en el presente estudio, indican que a medida que aumenta el RCS aumenta el LIP, en consecuencia el Grupo 1 posee un LIP de 375,03 días, por el contrario, el LIP del Grupo 4 se extiende a 389,54 días (Cuadro 4).

\section{CONCLUSIONES}

En base a los resultados obtenidos, analizados y discutidos en el presente estudio, se concluye que se rechaza la hipótesis planteada, según lo cual la época de parto y el recuento de células somáticas (RCS) no influyen sobre los rendimientos productivos y reproductivos para la raza Overo Colorado.

La época de parto, es un factor que debe ser considerado, debido a su influencia en la producción lo cual afecta directamente a la rentabilidad económica del sistema lechero. En el rubro lechero es importante tener un parto al año, bajo condiciones de pastoreo la época de parto se vuelve un factor preponderante, debido a que es ideal ajustar los requerimientos alimenticios del animal a la curva de producción de la pradera, por ello es fundamental identificar los factores que influyen sobre la reproducción del rebaño.

La presencia de mastitis en el rebaño eleva los RCS, lo cual provoca que la leche producida sea castigada por la industria y obtenga un menor precio. A mayor RCS, la pérdida productiva es mayor, lo cual se debe a la disminución en la cantidad de litros y calidad de la leche.

\section{REFERENCIAS}

ALIAGA, A. 2004. Se abrieron los registros de la raza por dos años. Overo Colorado. Profo Overos Colorados, ANACOC, Chile.

ALT, A. 2005. Análisis técnico económico de sistemas de producción de leche en base a la raza Overo Colorado en la $\mathrm{X}^{\circ}$ Región. Tesis Med. Vet., Universidad Austral de Chile. 49 p.

BUTENDIECK, N. 1997. Células somáticas, mastitis y calidad de leche. En: Calidad de leche e interpretación de resultados de laboratorio. Curso - Taller. INIA Carillanca Serie $\mathrm{N}^{\circ} 62$, Temuco, Chile, pp. 15-32.

CARRILLO, B., VIDAL, C. 2002. Análisis y aplicación de los esquemas de pago de la leche actualmente vigentes en la X región. En: Curso de mercados y gestión económica de la producción de leche. Fundación Chile. Ministerios de Agricultura. Santiago, pp. 1-11.

CONSORCIO LECHERO. 2013. Sólidos en leche. Tecnoláctea. http://www.consorciolechero.cl/tecnolactea/main-solidos-en-leche/?materia=5 (acceso, 15.12.2013).

CÓRDOVA, A., CóRDOVA, C., CÓRDOVA, M., SALTIJERAL, J., RUIZ, C., XOLALPA, V., CORTÉS, S., GUERRA, J. 2008. Efecto de la mastitis y el estrés sobre la reproducción de la vaca. Revista Veterinaria 19(2): 161-166.

FOOD AND AGRICULTURE ORGANIZATION (FAO). 2007. The State of the World's Animal Genetic Resources for Food and Agriculture, Roma.

FRICKE, P. 2009. Estrategias agresivas de manejo para mejorar la eficiencia reproductiva de vacas lecheras en lactancia. En: Avances en reproducción bovina. La reproducción y su consecuencia: la eficiencia reproductiva, Jornada Cooprinsem, Osorno, Chile. 
GASQUE, R. 2008. Enciclopedia bovina. Universidad Nacional Autónoma de México, Facultad de Medicina Veterinaria y Zootecnia, México.

GONZÁLEZ, H. 2008. Factores que afectan la productividad de los Sistemas pastoriles de producción de leche. Circular de Extensión Técnico Ganadera $N^{\circ} 34$, Universidad de Chile, Santiago.

GONZÁLEZ, H., MAGOFKE, J. 2007. Comportamiento de diferentes líneas de ganado Holstein en sistemas de producción basado en pastoreo. Universidad de Chile, Santiago.

HAENLEIN, G.F.W., SCHULTZ, L.H., HANSEN, L.R. 1968. Relation of Milk Fat-Depressing Rations and Subclinical Mastitis to Milk Proteins. Journal of Dairy Science 51(4), 535-542.

HAZARD, S. 1997. Calidad de la leche. Variación de la composición de la leche. En: Curso taller Calidad de Leche e Interpretación de Resultados de Laboratorio. INIA Carillanca Serie $N^{\circ} 62$. Temuco, Chile, pp. 33-44.

KLEIN, F. 2003. Utilización de praderas y nutrición de vacas a pastoreo. En: Teuber, K., Uribe, H., Opazo, L. (Eds.), Seminario hagamos de la lechería un mejor negocio. Serie actas INIA N$^{\circ} 24$, Osorno, Chile, pp. 43-55.

LATRILLE, L. 1985. La curva de lactancia. Algunos factores que afectan la cantidad y composición de la leche. Las reservas corporales al parto. La predicción del consumo voluntario. Alimentación de bovinos para producción de leche y carne. Instituto de Producción Animal. Universidad Austral de Chile, Valdivia.

MANTEROLA, H. 2007. Manejo nutricional y composición de la leche. El desafío e incrementar los sólidos totales en la leche. Una necesidad de corto plazo. Circular de Extensión Técnico Ganadera $N^{\circ} 33$, Universidad de Chile, Santiago.
NATZKE, R.P., SCHULTZ, L.H., BARR, G.R., HOLTMANN, W.B. 1965. Variation in mastitis screening test and milk composition of udders quarters under normal condition and following omission of a milking. Journal of Dairy Science 48(4): 1295-1304.

OFICINA DE ESTUDIOS Y POLÍTICAS AGRARIAS (ODEPA). 2014. Región de Los Lagos. Información regional 2014. ODEPA, Santiago.

PEDRAZA, C., MANSILLA, A., FAJARDO, P., AGÜERO, H. 2000. Cambios en la producción y composición láctea por efecto del incremento de células somáticas en leche de vacas. Agricultura Técnica 60: 251-258.

PÉREZ, L. 2003. Estudio de algunos factores no genéticos que afectan la producción y composición de la leche en un rebaño de pariciones biestacionales en la Décima Región. Tesis Ing. Agr., Universidad Austral de Chile. 143 p.

PHILPOT, N., NICKERSON, S. 2000. Winning the Fight Against Mastitis. Westfalia Surge, Inc., Napervile, Illinois.

URIBE, H., LANUZA, F. 2006. Reproducción. INIA Remehue, Boletín INIA N ${ }^{\circ} 148$, Osorno, Chile.

URIBE, H., SMULDERS, J. 2004. Estimación de parámetros y tendencias fenotípicas, ambientales y genéticas para características de producción de leche en bovinos Overos Colorados. INIA Remehue, Osorno, Chile.

VALDERRAMA, X. 2011. Proyecto mejoramiento de la composición de la leche mediante el manejo de la alimentación. Producción predial. Informes finales proyectos FIA, Chile.

WATTIAUX, M. 1999. Manejo de la eficiencia reproductiva. Esenciales Lecheras: Babcock Institute for International Dairy Research and Development. Universidad Wisconsin, Madison. 